\title{
SVM classifier fusion using belief functions: application to hyperspectral data classification
}

\author{
Marie Lachaize ${ }^{1,2}$, Sylvie Le Hégarat-Mascle ${ }^{1}$, Emanuel Aldea ${ }^{1}$, Aude Maitrot ${ }^{2}$ \\ and Roger Reynaud ${ }^{1}$ \\ ${ }^{1}$ SATIE, Université Paris-Sud, Université Paris-Saclay, 91405 Orsay, France, \\ ${ }^{2}$ VEOLIA RECHERCHE \& INNOVATION, 291 av Dreyfous Ducas, Limay, France \\ ${ }^{1}$ firstname.lastname@u-psud.fr, ${ }^{2}$ firstname.lastname@veolia.com
}

\begin{abstract}
Hyperspectral imagery is a powerful source of information for recognition problems in a variety of fields. However, the resulting data volume is a challenge for classification methods especially considering industrial context requirements. Support Vector Machines (SVMs), commonly used classifiers for hyperspectral data, are originally suited for binary problems. Basing our study on [12] bbas allocation for binary classifiers, we investigate different strategies to combine two-class SVMs and tackle the multiclass problem. We evaluate the use of belief functions regarding the matter of SVM fusion with hyperspectral data for a waste sorting industrial application. We specifically highlight two possible ways of building a fast multi-class classifier using the belief functions framework that takes into account the process uncertainties and can use different information sources such as complementary spectra features.
\end{abstract}

Keywords: Hyperspectral classification, Belief Function Theory, waste sorting.

\section{Introduction}

Hyperspectral imaging acquires an almost continuous sampling of the spectral data in each pixel of a scene. The large number of narrow and contiguous bands allows us to detect even minor variations in a spectrum and provides a significant advantage for the distinction of different materials (natural or artificial). Hyperspectral imaging popularity has thus increased during the last decade, due both to the increase in computing power (that allows us to process these voluminous data) and to the need of separation of increasingly specific classes, e.g. different kinds of polymers for industrial application or different mineralogical compositions of surfaces for remote sensing applications. The acquisition of numerous wavebands enables the identification of fine classes belonging to various fields: atmosphere analysis, ecosystems monitoring, military applications and industrial applications (e.g. [5]).

Recently, hyperspectral imaging industrial applications appeared in waste sorting field. This latter needs new automated processes to improve the working conditions and decrease the cost of recycled materials. This requires sensors able 
to discriminate among waste materials as close (in terms of spectral response) as different kinds of plastics or different fibrous materials. Then, some efficient classification methods should be defined to use the full potential of hyperspectral data in this industrial context: methods adapted to waste material classes, robust to significant object occlusion (objects stacked on one another), and fast enough to match the waste flow.

Hyperspectral imagery provides detailed spectral information for each pixel of the picture. This information richness comes with an obvious drawback: the huge amount of data to process. Indeed, it involves significant computing resources (time, memory) that may be an issue in an industrial context. In addition to spectral information, several works also propose to include spatial information in their classification process, e.g. [13]. However, in this study, we focus on blind classification, i.e. classification of each pixel independently (not taking into account the neighbouring pixels that provide the spatial information). For our industrial application, it is a first step towards a classification process at object level which couples blind classification and spatial segmentation using others sensors.

Support vector machines (SVMs) are classically used to meet the challenge of hyperspectral classification, see $[8,10,7]$ for instance. Basically, they project data into a higher dimensional space in which classes can be separated using (optimal) hyperplanes. SVMs are widely used in a probabilistic framework (e.g. through logistic regression). However, probabilities are not able to distinguish between uncertainty (in decision process) due to ambiguity, e.g. because of overlapping between classes, and uncertainty due to imprecision, e.g. because of low number of samples for some score values during the SVM training and calibration processes. Thus, [12] has extended several classical regressions used for SVM binary classifiers to the Belief Function Theory framework.

As SVMs are well suited for 2-class problems, several strategies have been proposed to address the multiclass case. Classical approaches are: (i) the oneversus-one strategy, where a classifier is trained on each class pair and (ii) the one-versus-all strategy, where a classifier is trained to distinguish one class against all the others. For each strategy, the merging of these 2-class classifiers is an important issue. In this study, we propose and evaluate three strategies to combine binary SVM outputs in the BFT framework. We also show that the use of belief functions allows us to combine the information contained into different input data derived from the whole spectrum data, and that it is an efficient way to deal with the complexity involved by the high dimensionality of the hyperspectral data.

\section{Strategies from binary to multiclass belief functions}

Addressing a multiclass problem from binary SVMs (one-versus-one or oneversus-all), we have to combine their binary decisions while taking into account the imprecision of each SVM (classes overlapping, number of samples, ... ). We choose to perform this combination in BFT framework considering SVM outputs 
as logical sources. The way an observed score generates a basic belief assignment (bba) is defined by the calibration step proposed by [12]. It is briefly recalled in Appendix. Then, in the following, we assume that each cluster of SVM produces as many bbas as there are SVMs in the cluster, and that each bba represents our belief in each of the two classes handled by the considered SVM as well as the ignorance left by this classifier.

Notation are as follows. For any one-versus-one SVM dealing with the pair of classes $\omega_{j}$ and $\omega_{k}, \Omega_{j, k}^{b}$ denotes the associated discernement frame and $m^{\Omega_{j, k}^{b}}$ the bba derived from calibration. For any one-versus-all SVM dealing with the pair of classes $\omega_{j}$ and its complementary $\bar{\omega}_{j}, \Omega_{j}^{b}$ denotes the associated discernement frame and $m^{\Omega_{j}^{b}}$ the bba derived from calibration. In both cases, the upperscript $b$ recalls that SVM was binary and the cardinality of $\Omega_{j, k}^{b}$ or $\Omega_{j}^{b}$ is 2 . Then, the transition from binary classification to a multiclass one implies a change of discernment frame from $\Omega_{j, k}^{b}$ or $\Omega_{j}^{b}$ to $\Omega=\left\{\omega_{1}, \ldots, \omega_{n}\right\}$, the multiclass set.

\subsection{Case of one-versus-one SVMs}

In the one-versus-one strategy, $\Omega_{j, k}^{b}=\left\{\omega_{j}, \omega_{k}\right\}$ and $m^{\Omega_{j, k}^{b}}$ is interpreted as the result of a conditioning of a multiclass bba $m^{\Omega}$ on $\Omega_{j, k}^{b}$, noted $m^{\Omega}\left[\Omega_{j, k}^{b}\right]$. Then, from these conditioned estimations of the bba, [9] proposes to derive $m^{\Omega}$ by solving the optimization problem:

$$
\min _{m^{\Omega}} \sum_{k>j} \sum_{\emptyset \neq A \subseteq \Omega}\left(m^{\Omega}\left[\Omega_{j, k}^{b}\right](A)-m^{\Omega_{j, k}^{b}}(A)\left(1-m^{\Omega}\left[\Omega_{j, k}^{b}\right](\emptyset)\right)\right)^{2},
$$

under the constraints: (i) $m^{\Omega}(A) \geq 0, \forall A \in 2^{\Omega}$, (ii) $m^{\Omega}(\emptyset)=0$ and (iii) $\sum_{A \in 2^{\Omega}} m^{\Omega}(A)=1$. In Eq.(1), the factor $1-m^{\Omega}\left[\Omega_{j, k}^{b}\right](\emptyset)$ is due to the fact that $m^{\Omega_{j, k}^{b}}$ is a normal bba which may not be the case for $m^{\Omega}\left[\Omega_{j, k}^{b}\right]$. To solve such a constrained system, we have noticed that it can also be written as a matrix system $\min _{\mathbf{X}}|\mathbf{A X}-\mathbf{B}|^{2}$.

In this study, we propose an alternative to derive $m^{\Omega}$ that consists in simply performing a deconditioning of every bba $m^{\Omega_{j, k}^{b}}$ on the frame of discernment $\Omega$ and then combining the deconditioned bbas $m^{\Omega_{j, k}^{b} \Uparrow \Omega}$ using a conjunctive rule: denoting $B=\bar{A}$ the complementary in $\Omega$ of hypothesis $A$,

with

$$
m^{\Omega}(A)=\bigcirc_{(j, k) \in[1, n]^{2}} m^{\Omega_{j, k}^{b} \Uparrow \Omega}(A), \forall A \in 2^{\Omega},
$$

$$
m^{\Omega_{j, k}^{b} \Uparrow \Omega}\left(A \cup \bar{\Omega}_{j, k}\right)=m^{\Omega_{j, k}^{b}}(A), \forall A \in\left\{\omega_{j}, \omega_{k},\left\{\omega_{j}, \omega_{k}\right\}\right\} .
$$

This second way is much simpler than the first one and it is theoretically founded on the independence between the binary SVM outputs. Now, it could provide an interesting approximation of the solution and even, it outperforms the optimization proposed by [9] in our application case. Therefore, on this latter at least, there are only advantages in using the proposed alternative. 


\subsection{Case of one-versus-all SVMs}

In the one-versus-all strategy, the binary discernment frames $\Omega_{j}^{b}$ are some coarsenings of discernment frame $\Omega$, or equivalently $\Omega$ is a common refinement of the different $\Omega_{j}^{b}=\left\{\omega_{j}, \bar{\omega}_{j}\right\}$. Then, the bbas defined on $\Omega_{j}^{b}$ are refined on $\Omega$ using classical refinement operator, before combination using Dempster's rule:

$$
m^{\Omega}(A)=\oplus_{j \in[1, n]} m^{\Omega_{j}^{b} \uparrow \Omega}(A), \forall A \in 2^{\Omega},
$$

with

$$
m^{\Omega_{j}^{b} \uparrow \Omega}(A)=m^{\Omega_{j}^{b}}(A), \forall A \in\left\{\omega_{j}, \bar{\omega}_{j}, \Omega\right\} .
$$

Here, we use the orthogonal sum instead of the conjunctive combination rule since, due to the existence of singleton focal elements and to the high number of combinations (as much as the number of classes), conflict becomes very important even in standard cases.

\subsection{Case of hybrid strategy}

Each of the two previous strategies handles a given kind of binary SVMs, either one-versus-one or one-versus-all. Each of these strategies has some advantages: better separability of the classes for the one-versus-one SVMs, simplicity and speed for the one-versus-all SVMs. Then, in order to benefit from both advantages, we propose a hybrid strategy that handles SVMs belonging to both kinds of binary SVMs.

The proposed solution is based on some metaknowledge on the classes used to choose the considered SVMs. Let us first present the particular case of a hierarchical strategy through a toy example with only 4 classes: $\Omega=\left\{\omega_{1}, \omega_{2}, \omega_{3}, \omega_{4}\right\}$, and assume $\omega_{3}$ and $\omega_{4}$ classes are difficult to separate. According to the proposed strategy, one will focus on most performing SVMs, i.e. SVMs ' $\omega_{1}$ against $\left\{\omega_{2}, \omega_{3}, \omega_{4}\right\}$ ', ' $\omega_{2}$ against $\left\{\omega_{1}, \omega_{3}, \omega_{4}\right\}$ ', ' $\left\{\omega_{3}, \omega_{4}\right\}$ against $\left\{\omega_{1}, \omega_{2}\right\}$ ' and ' $\omega_{3}$ against $\omega_{4}$ '. In a more general way, the classes of $\Omega$ are 'wisely' grouped to form a new coarsened discernment frame on which the one-versus-all approach performs well ('good' enough class separability, such as for $\left\{\omega_{1}, \omega_{2},\left\{\omega_{3}, \omega_{4}\right\}\right\}$ in the example). Then, the considered classifiers are: the one-versus-all SVMs for classes corresponding to singleton hypotheses of the coarsened discernement frame (i.e. some of them are compound classes, e.g. hypothesis $\left\{\omega_{3}, \omega_{4}\right\}$ in the exemple) and the one-versus-one SVMs for classes belonging to a coumpound class of the coarsened discernment frame (e.g. SVM $\omega_{3}$ versus $\omega_{4}$ in the example). The bbas derived from outputs of previously cited SVMs that have been either refined or deconditionned on $\Omega$ are combined using conjunctive rule (or Dempster's orthogonal rule).

This hierarchical strategy appears as a compromise in terms of number of used (and thus trained) classifiers: if $n=|\Omega|$, with the pure one-versusone strategy, we have to consider $\left(\begin{array}{l}n \\ 2\end{array}\right)=\frac{n(n-1)}{2}$ classifiers, with the pure oneversus-all strategy, we have to consider $n$ classifiers and with the hierarchical strategy involving $l$ groups of $n_{i}$ indistinguishable classes, we have to consider 
$\left.n-\sum_{i=1}^{l}\left(n_{i}-1\right)\right)$ one-versus-all classifiers and $\sum_{i=1}^{l} \frac{n_{i}\left(n_{i}-1\right)}{2}$ one-versus-one classifiers.

With respect to the hierarchical strategy, the hybrid strategy can in addition consider few other classifiers in order to increase the redundancy between classifiers and then provide more robust results. However, this number of additional classifiers should remain low to keep an interest in terms of complexity.

Finally, if the derivation of the metaknowledge is a subject beyond the scope of this article (here we simply assume that it can be either known a priori or learnt from training samples), let us underline that it is the crucial point for the proposed hybrid strategy since it allows us to add prior information (metaknowledge) that should be carefully chosen.

\subsection{Decision}

Independently of the strategy used, at the end, we have a bba resulting from the combination of the information pieces provided by different binary SVMs taking into account their own features (in particular the learning step conditions and results). From this bba, we can take a decision or, as seen in the experimental part, combine it with other bba(s) and then take the decision.

The belief function framework offers many possibilities for decision making [3]. Among them, pessimistic and optimistic strategies consist in maximizing, over the singleton hypotheses, the belief or plausibility function, respectively. A decision according to the pignistic probability provides intermediate results.

Here, specific to the one-versus-one strategy based optimization [9], we also test the following criterion: the decided singleton class $\omega_{i}$ maximizes the conflict generated by conditioning on binary subsets not including $\omega_{i}$ :

$$
\omega_{i}=\arg \max _{i} \sum_{j, k \neq i} m^{\Omega}\left[\Omega_{j, k}^{b}\right](\emptyset) .
$$

\section{Experiments}

\subsection{Industrial context and data preprocessing}

We applied the proposed classification strategies to waste sorting. Despite the use of some sorting components exploiting the mechanical properties of the waste materials in order to separate them, this industrial application has still several issues such as the automatic identification of some resembling materials or the detection of some 'intruders' in a set of similar wastes (e.g. paper, cardboard and plastic waste). For such purposes, the hyperspectral sensor appears relevant since it provides some information about the nature of the material itself that should help us to discriminate among different fibrous materials and polymers with high throughput and a reliability and robustness suitable for an industrial context.

As with any classification method, the performance of a SVM classifier strongly depends on the input data. Classical preprocessing on the spectrum involves a 
filtering and derivation at different orders. Specifically, the Savitsky-Golay filter is widely used for hyperspectral data analysis $[6,11]$. This filter fits a low degree polynomial on data within a sliding window having fixed size. It allows us to smooth the data and to compute the derivatives from the fitted polynoms. The fact of considering different derivative orders (typically 0,1 and 2) appears all the more justified since, for classification, not the whole spectrum is considered but only some selected features, in order to reduce both the data complexity and the correlation between the bands. Then, a classical way is to perform a Principal Component Analysis (PCA) on the filtered spectra, e.g. [2,1]. In our case, the number of selected components is set to represent $99 \%$ of the information. It varies between 3 to about 20 whereas the whole spectrum dimensionality was about 275 .

In summary, preprocessing involves the computation of different derivative orders $(0,1$ and 2$)$ of the spectrum by the Savitsky-Golay filter and then, for each of these derivatives, the computation of the PCA that provides the input data for the SVM classifiers. In the following, these input data are denoted $S_{0}$, $S_{1}$ and $S_{2}$ where subscript denotes the derivation order. The results obtained using these inputs will be compared in terms of classification performance. We also propose to use them as different logical sources so that, we combine the multiclass (defined on $\Omega$ ) bbas derived for given input data $\left(S_{0}, S_{1}\right.$ and $S_{2}$ ). Assuming that the PCA process makes input data cognitively independent, bba combination will be done through the conjunctive rule.

\section{$3.2 \quad$ Experimental results}

The sample sets used for these experiments have been collected in the Veolia laboratories on a hyperspectral sensor (whose spectra contains about 275 wavelengths) via specimen boards with small material samples: four boards called Paper, Plastic1, Plastic2a, Plastic2b. Samples are divided in 9 classes, namely 7 polymers classes (not listed here for paper shortness) and 2 fibrous classes (paper and cardboard). From specimen boards, three different datasets were extracted. The first one, called training set, has 1000 samples per class and is used for SVM training. The second one, called calibration set, has 200 samples per class and is used for bba calibration. The last one has 1000 samples per class and is used for test and performance estimation. In addition to this test dataset, another board, exclusively used for testing and called Superposition, presents real objects stacked on top of each other to provide more realistic conditions.

Then, the training set allows for the estimation of each SVM classifier parameters, determined by cross validation and grid search, using Gaussian kernels. The calibration set allows for the estimation of sigmoid parameters and contour function defined for any score value (see Appendix for details). It also allows us to determine the classes to group for the hybrid strategy. Then, using the test set and the Superposition board, the first analysis (not presented here) puts forward some complementarity of classification performance for the input data $S_{1}$ and $S_{2}$, in particular for the 'difficult' pixels such as those present in the shadows or pixels corresponding to the superposition of two objects. The initial 


\begin{tabular}{|c|c|c|c|c|c|}
\hline Strategy $\rightarrow$ & \multicolumn{3}{|c|}{ one-versus-one } & one-versus-all & hybrid \\
\hline$\downarrow$ Boards & score vote & optimization & deconditioning & & \\
\hline Paper & 95.3 & 95.2 & $\mathbf{9 5 . 5}$ & 91.6 & 94.1 \\
Plastic1 & 90.2 & 87.3 & $\mathbf{9 1 . 4}$ & 85.2 & 91.0 \\
Plastic2a & 79.1 & 83.7 & $\mathbf{8 4 . 1}$ & 77.5 & 79.5 \\
Plastic2b & 87.2 & 89.3 & $\mathbf{8 9 . 7}$ & 83.9 & 87.8 \\
Superposition & 82.8 & 88.0 & $\mathbf{8 8 . 4}$ & 79.5 & 80.1 \\
\hline whole data & 86.9 & 88.8 & $\mathbf{8 9 . 8}$ & 83.5 & 86.5 \\
\hline
\end{tabular}

Table 1: Correct classification rate (in \%) for the 5 test sample boards (each one having 13500 pixels). Results are given for $S_{1}$ source, considering different strategies and decisions for the one-versus-one strategy.

analysis also revealed that the $S_{0}$ input data provides results of little interest (low performance and lower complementarity) so that it has not been considered in the results presented further.

\subsection{Comparison of the different strategies}

The comparison of the different strategy is presented here in the case of $S_{1}$ data that prompts better results than $S_{0}$ or $S_{2}$. Considering $S_{1}$, the hybrid strategy was instanciated introducing two coarse classes: one grouping paper and cardboard and another grouping two classes of polymers (among the 7). A supplementary one-versus-one classifier is also considered to remove ambiguities between two other classes of polymers.

Classification results are analyzed in the perspective between comparison of (i) different multi-class strategies (one-versus-one, one-versus-all, hybrid), (ii) different decision making processes for the one-vs-one strategy. Quantitative results, computed on the whole datasets, are shown in Tab. 1. Our main conclusions are:

- In the case of the one-versus-one strategy, solving Eq. (1), the computation time increases dramatically with the number of classes (factor about 200 with the one-vs-all strategy computation time). Performing deconditioning (Eq. (2)) as proposed provides slightly better results for a much lower computation time.

- Comparison with classic decision rules, either score voting (shown in Tab. 1) or probabilistic decision (not shown) shows that the optimistic decision on bba obtained using either optimization or deconditioning (Eq. (2)) is better in every cases.

- The one-vs-one strategy always outperforms the one-vs-all strategy and the hybrid one, which is a standard result due to the better separability of the classes and much higher number of considered classifiers.

- The hybrid strategy shows intermediate results between the one-vs-one strategy and the one-vs-all one: depending on the considered test dataset, the improvement relatively to the one-vs-all strategy varies between $0.6 \%$ and $5.8 \%$. 

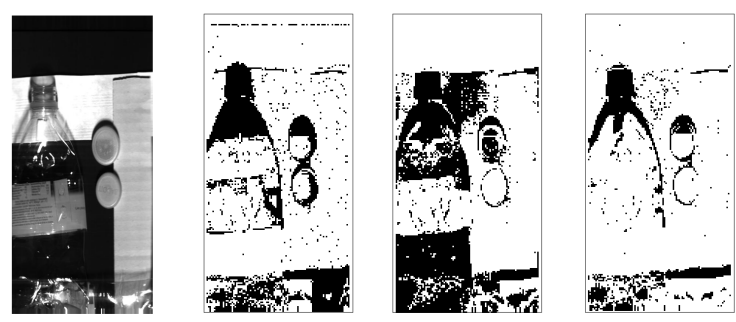

Fig. 1: Example of the fusion impact on classification rate (Superposition board). From left to right: test board image, binary representations of the well classified pixels (in white) considering $S_{1}, S_{2}$ and their evidential fusion, respectively.

\begin{tabular}{|c|c|c|c|c|c||c|}
\hline Boards $\rightarrow$ & Paper & Plastic1 & Plastic2a & Plastic2b & Superposition & whole data \\
\hline one-versus-one & 93.3 & $\mathbf{9 3 . 4}$ & $\mathbf{8 2 . 2}$ & $\mathbf{8 7 . 9}$ & $\mathbf{8 9 . 9}$ & $\mathbf{8 9 . 3}$ \\
\hline one-versus-all & 92.3 & 91.8 & 80.1 & 87.1 & 87.3 & 87.7 \\
\hline hybrid & $\mathbf{9 5 . 9}$ & 92.2 & 78.4 & 87.3 & 86.1 & 88.0 \\
\hline
\end{tabular}

Table 2: Correct classification rate (in \%) for the 5 test sample boards. Results are given for the $S_{1} \& S_{2}$ fusion, considering different strategies.

\subsection{Combination of sources $S_{1}$ and $S_{2}$}

In this subpart, classification results are analyzed versus different input data, namely both $S_{1}$ and $S_{2}$ (combined by fusion) or only $S_{1}$ (shown in Tab. 1). Quantitative results are shown on Tab. 2. Our main conclusions are:

- The fusion of $S_{1}$ and $S_{2}$ sources provides no or low improvement compared to the one-vs-one strategy, however the complementarity is very beneficial to the one-versus-all strategy and narrows the gap between the classification results of the two strategies (from $6.3 \%$ to $1.5 \%$ on the whole dataset).

- Figure 1 presents the Superposition results illustrating that the fusion improves particularly the classification of the difficult pixels, such as at the top of the bottle and the caps stacked on paper.

- The hybrid strategy still provides intermediate results between one-versusone strategy and one-versus-all one, but the interest relatively to the oneversus-all is reduced (relatively to the case of the only- $S_{1}$ data) due to the high improvement of performance of one-versus-all strategy provided by fusion.

\section{Conclusion and perspectives}

This study has investigated the possibility in BFT to build a multiclass classifier which would be fast and efficient enough to be considered in the industrial 
context of waste sorting. We compare different ways to achieve it: using the deconditioning operator on bbas derived from one-vs-one classifiers, using the refinement operator on bbas derived from one-vs-all classifiers or a using hybrid strategy. According to our tests, using one-vs-one leads to better performance but requires more classifiers to train than using one-vs-all classifiers. Using optimization [9] to derive the multiclass bba from bbas derived from one-vs-one classifiers can be advantageously replaced by the proposed deconditioning and conjunctive combination in terms of computation time. Hybrid strategy seems a good compromise between pure one-vs-one and pure one-vs-all strategies, presenting both reasonable number of classifiers and interesting performance results. Combining multiclass bbas associated to different features of the hyperspectral spectra (different orders of derivative) enhances the classification results in a noticeable way.

A main perspective to our work is the automatic derivation of the metaknowledge on data used to build the hybrid strategy. We saw that the results provided by hybrid strategy are encouraging. However, the impact of the metaknowledge has to be investigated and this also indirectly raises the question of the calibration quality of the binary classifiers. Then, we also intend to investigate some evidential criteria that will allow us to analyze training calibration sets, for instance to group automatically some classes and make the most of the hybrid strategy.

\section{Appendix: Evidential calibration}

Handling binary classifiers, the discernment frame is $\Omega^{b}=\{\{0\},\{1\}\}$. Then, for a given SVM having its own features in terms of number of samples, learning step performance, we aim at defining a belief function for each score that reflects the confidence we may have in each class. Indeed, this belief function will be used for forecasting taking into account the whole training set specificities. Explicitly, for each score $s$, the mass function, denoted $m$, is derived from the contour function: $\omega \rightarrow p l_{X}(\omega, s)$, where $\omega=P(y=1 \mid s)$ (note that, $\omega$ is not a class but a probability).

To build the contour function on $\omega,[12]$ uses the idea behind the logistic regression: approximating the probability $P(y=1 \mid s)$ by a sigmoid $\operatorname{sig}_{s}(\theta)=$ $\left[1+\exp \left(\theta_{0}+\theta_{1} s\right)\right]^{-1}$, where the parameter $\hat{\theta}=\left(\theta_{0}, \theta_{1}\right)$ is determined by maximizing the likelihood function $L_{X}(\theta)$ over the training set $X=\left\{\left(s_{1}, y_{1}\right), \ldots,\left(s_{N}, y_{N}\right)\right\}$ where, for each sample number $i, s_{i} \in \mathbb{R}$ is the score given by the considered classifier and $y_{i} \in\{0,1\}$ is its true label. Then, the contour function of interest is drawn for a given value of score. It derives from the $2 \mathrm{D}$ function plotting the relative value of the likelihood function $\frac{L_{X}(\theta)}{L_{X}(\hat{\theta})}$ versus $\theta=\left(\theta_{0}, \theta_{1}\right)$.Then, for any given pair $(s, \omega)$, the set of $\theta$ (i.e. $\left.\operatorname{sig}_{s}^{-1}(\omega)\right)$ values is a straight line in $\mathbb{R}^{2}$. Then, the contour function value can be determined as the maximum value over this straight line: 


$$
p l_{X}^{\Omega_{j, k}^{b}}(\omega)= \begin{cases}0 & \text { if } \omega \in\{0,1\} \\ \sup _{s i g_{s}^{-1}(\omega)} \frac{L_{X}(\theta)}{L_{X}(\hat{\theta})} & \text { otherwise }\end{cases}
$$

with $L_{X}(\theta)=\prod_{i=1}^{N} p_{i}^{y_{i}}\left(1-p_{i}\right)^{1-y_{i}}$ where $p_{i}=\frac{1}{1+\exp \left(\theta_{0}+\theta_{1} s_{i}\right)}$. Finally, from each $p l_{X}^{\Omega_{j, k}^{b}}$, the corresponding mass function $m^{\Omega_{j, k}^{b}}$ on binary discernment frame $\Omega_{j, k}^{b}$ is derived using the 'likelihood based' belief function for statistical inference approach proposed by Shafer and further justified by Denœux [4].

\section{References}

1. R. Cavalli, G. Licciardi, and J. Chanussot. Archaeological structures using nonlinear principal component analysis applied to airborne hyperspectral image. IEEE J. of Selected Topics in Applied Earth Observations and Remote Sensing, 6(2):659669, 2013.

2. G. Chen and S.-E. Qian. Denoising of hyperspectral imagery using principal component analysis and wavelet shrinkage. IEEE Trans. on Geoscience and Remote Sensing, 49(3):973-980, 2011.

3. T. Denoeux. Analysis of evidence-theoretic decision rules for pattern classification. Pattern recognition, 30(7):1095-1107, 1997.

4. T. Denœux. Likelihood-based belief function: justification and some extensions to low-quality data. Int. J. of Approximate Reasoning, 55(7):1535-1547, 2014.

5. H. Grahn and P. Geladi. Techniques and applications of hyperspectral image analysis. John Wiley \& Sons, 2007.

6. R. L. King, C. Ruffin, F. LaMastus, and D. Shaw. The analysis of hyperspectral data using savitzky-golay filtering-practical issues. 2. In Proceedings of IGARSS'99, volume 1, pages 398-400. IEEE, 1999.

7. B.-C. Kuo, H.-H. Ho, C.-H. Li, C.-C. Hung, and J.-S. Taur. A kernel-based feature selection method for svm with rbf kernel for hyperspectral image classification. IEEE J. of Selected Topics in Applied Earth Observations and Remote Sensing, 7(1):317-326, 2014.

8. F. Melgani and L. Bruzzone. Classification of hyperspectral remote sensing images with support vector machines. IEEE Trans. on Geoscience and Remote Sensing, 42(8):1778-1790, 2004.

9. B. Quost, T. Denœux, and M.-H. Masson. Pairwise classifier combination using belief functions. Pattern Recognition Letters, 28(5):644-653, 2007.

10. S. Samiappan, S. Prasad, and L. M. Bruce. Non-uniform random feature selection and kernel density scoring with svm based ensemble classification for hyperspectral image analysis. IEEE J. of Selected Topics in Applied Earth Observations and Remote Sensing, 6(2):792-800, 2013.

11. C. Vaiphasa. Consideration of smoothing techniques for hyperspectral remote sensing. ISPRS J. of Photogrammetry and Remote Sensing, 60(2):91-99, 2006.

12. P. Xu, F. Davoine, H. Zha, and T. Denoeux. Evidential calibration of binary svm classifiers. Int. J. of Approximate Reasoning, 72:55-70, 2016.

13. J.-H. Yang, L.-G. Wang, and J.-X. Qian. Hyperspectral image classification based on spatial and spectral features and sparse representation. Applied Geophysics, 11(4):489-499, 2014. 